\title{
Diacronie
}

Studi di Storia Contemporanea

$N^{\circ} 22,2 \mid 2015$

Costruire

\section{Lorenzo Ettorre, Il Pci e il Concilio Vaticano II. Dal partito dei cattolici al cattolicesimo}

\section{Giorgio Vecchio}

\section{(2) OpenEdition \\ Journals}

Edizione digitale

URL: http://journals.openedition.org/diacronie/2118

DOI: 10.4000/diacronie.2118

ISSN: 2038-0925

Editore

Association culturelle Diacronie

Notizia bibliografica digitale

Giorgio Vecchio, «Lorenzo Ettorre, II Pci e il Concilio Vaticano II. Dal partito dei cattolici al cattolicesimo », Diacronie [Online], № 22, 2 | 2015, documento 19, online dal 01 juin 2015, consultato il 10 décembre 2020. URL : http://journals.openedition.org/diacronie/2118; DOI : https://doi.org/10.4000/diacronie. 2118 


\section{RECENSIONE:}

\section{Lorenzo ETTORRE, Il Pci e il Concilio Vaticano II. Dal partito dei cattolici al cattolicesimo, Roma, Studium, 2014, 188 pp.}

a cura di Giorgio VECCHIO *

Questo agile testo - opera iniziale di un valido giovane - è nettamente diviso in due parti. Nella prima l'autore propone un sintetico, ma efficace, panorama della "questione cattolica" così come impostata dai comunisti, a partire dalle note riflessioni di Gramsci e dalle successive acquisizioni di Togliatti. Si tratta di un argomento molto studiato, sul quale è difficile offrire novità rilevanti. Ettorre ha cercato di ovviare a questa situazione sistemando in modo organico le acquisizioni storiografiche disponibili e aggiungendo, qua e là, materiale tratto dall'archivio del PCI e relativo all'attività e alla corrispondenza di militanti e dirigenti locali. In tal modo egli stabilisce un punto fermo nella propria valutazione, ovvero sottolinea il precario equilibrio stabilitosi tra le direttive del vertice e i sentimenti della base, la quale in più di un caso non comprende le direttive ricevute, in quanto mantiene riserve di natura laicistica e anticlericale. Ciò dipende anche dal fatto di trovarsi direttamente a confronto con i parroci, in un clima quanto mai teso e conflittuale ${ }^{1}$.

In ogni caso, sono confermati i limiti dell'analisi della dirigenza comunista Togliatti e Longo compresi - , che fatica molto a comprendere l'essenza della fede religiosa, anche a proposito dei legami intercorrenti tra i fedeli e l'episcopato o il papa, nei quali concorrevano (e concorrono) fattori non riducibili agli schemi strettamente politici. Ettorre lo mette bene in luce riprendendo peraltro osservazioni già mosse da

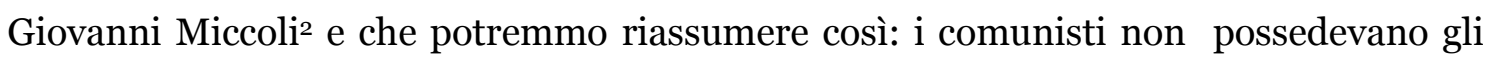
strumenti culturali per comprendere che il legame tra base e vertice nella Chiesa

\footnotetext{
${ }^{1}$ ETTORRE, Lorenzo, Il Pci e il Concilio Vaticano II. Dal partito dei cattolici al cattolicesimo, Milano, Studium, 2014, pp. 50-54

2 Ibidem, pp. 42-43.
} 
dipendeva da spiegazioni extra-politiche, bensì religiose, di fede, di affetto. Dopo tutto, si potrebbe osservare, i fedeli si riferivano a Pio XII o al proprio vescovo con quello stesso groviglio di sentimenti di identificazione che gli stessi comunisti manifestavano nei confronti di Stalin o di Togliatti. In una prospettiva del genere la tendenza a privilegiare il rapporto con la DC rispetto a quello con la Chiesa e la speranza di inserire dei cunei tra fedeli e gerarchia non potevano portare da alcuna parte.

Su questa prima parte del libro meritano però di essere mosse un paio di osservazioni di più vasta portata. Anzitutto andrebbe discussa un'affermazione di Ettorre, secondo cui la campagna per la pace promossa tra la fine degli anni Quaranta e i primi anni Cinquanta fu uno dei «capolavori politici» di Togliatti ${ }^{3}$. Pare invece che quella mobilitazione fu più subita che guidata dal segretario comunista che, anzi, cercò di contenere le spinte di chi - come Emilio Sereni - avrebbe voluto impiegare in essa maggiori energie del partito. D'altra parte, quella campagna rientrava tra le direttive di Stalin e del Cominform - oltre che rispondere a un sentimento diffuso tra l'intera popolazione - e quindi era impossibile metterla da parte. Togliatti sostenne certamente il movimento dei partigiani della pace, ma senza investirvi più di tanto le energie personali e delegando ad altri (Pajetta, Sereni, poi Spano) il compito di seguirla e talvolta frenandone l'eccessivo entusiasmo. L'affermazione di Ettorre può essere interpretata nel senso che il "capolavoro" del segretario comunista fu quello di sostenere la campagna per la pace, ma senza farsene condizionare più di tanto.

In secondo luogo rimane qui assente - come in pressoché tutta la storiografia l'analisi delle conseguenze dello scontro tra Chiesa e regimi comunisti dell'Est che si svolse in quegli anni. La cosiddetta Chiesa del silenzio assurse a chiave di lettura fondamentale nel mondo cattolico per giudicare l'attendibilità delle proposte comuniste e sorprende dunque constatare che questo argomento sia per lo più dimenticato. Si trattava di un macigno gigantesco posto sulla strada di ogni possibile "dialogo", anche prescindendo da ogni altro tema, ideologico o politico che fosse. Basterebbe del resto sfogliare la stampa cattolica del tempo, a cominciare da «L'Osservatore Romano», per verificare quanta attenzione e passione fosse dedicata alle vicende dei vari Stepinac, Wyszyński, Mindszenty o Beran ${ }^{4}$.

\footnotetext{
3 Ibidem, pp. 66-67.

4 Per es. l'arresto del primate d'Ungheria ebbe un risalto enorme su «L'Osservatore Romano»: a partire dal 29 dicembre 1948 e fino circa alla metà di marzo del 1949, la prima pagina del quotidiano della S. Sede fu sempre dominata da titoli e articoli sull'argomento, con una sola eccezione in gennaio e pochissime in febbraio per dar risalto a interventi di Pio XII. Dal 19 giugno 1949 «L'Osservatore Romano» diede analogo risalto alla situazione della Chiesa cecoslovacca dopo l'arresto di mons. Beran. In confronto ebbe molto meno spazio sul giornale, nell'ottobre 1946, il processo di Zagabria contro mons. Stepinac.
} 
Ricordato che gli avvenimenti più clamorosi per la Chiesa in Europa orientale avvennero temporalmente dopo le elezioni italiane del 1948, rimane significativo il fatto che, all'indomani della bruciante sconfitta del 18 aprile, la grande maggioranza dei membri della direzione del PCI si riducesse a spiegare l'intervento della Chiesa nella campagna elettorale con la sola definizione di «terrorismo religioso», tanto da suscitare la delusione dello stesso Togliatti, il quale affermò esplicitamente:

La discussione non è stata buona. La critica dei risultati è stata troppo superficiale $\mathrm{e}$ non collegata con la necessaria autocritica. I compagni hanno dato un contributo insufficiente alla elaborazione della nostra linea politica. [...] Cattivo è stato l'intervento del compagno Pajetta: ci aspettavamo di più da lui, almeno una maggiore autocritica e se non altro credevamo ci risparmiasse questo suo atteggiamento di stanchezza e di insoddisfazione5.

Con un certo salto - in parte pure temporale, almeno come livello di approfondimento - la seconda parte del libro entra direttamente nell'argomento promesso dal titolo. In due capitoli Ettorre ci fa seguire il progressivo, ma irreversibile, mutamento dei giudizi comunisti su Giovanni XXIII e sulle novità da lui introdotte. Analizzando la stampa comunista - compresa quella «Rinascita» che, curiosamente, in questo libro viene sempre ribattezzata «La Rinascita» - Ettorre segnala come l’iniziale idea di una continuità tra Giovanni XXIII e Pio XII sia stata via via sostituita da un crescente entusiasmo per il "Papa buono", specialmente dopo l'udienza accordata alla figlia e al genero di Chruščëv e dopo la pubblicazione dell'enciclica Pacem in terris. Anche in questi passaggi, tuttavia, l'autore rileva come il giudizio dei militanti sembri rimanere "più chiuso e retrogrado" rispetto a quello dei dirigenti ${ }^{6}$. Tra i punti più interessanti colti qui da Ettorre sta anche il mutamento di valutazioni sulle aperture ecumeniche della Chiesa cattolica: inizialmente intese solo come strumento di guerra fredda (dal momento che la maggior parte dei regimi dell'Europa orientale avevano a che fare con cristiani ortodossi), esse vennero poi giudicate utili ai fini della distensione e della pace internazionale.

5 Fondazione Gramsci, Archivio del Partito Comunista, Direzione, Verbali, riunione del 26 aprile 1948. Nella discussione Ciufoli aveva descritto l'andamento delle elezioni in Puglia, affermando tra l'altro: «Potremo noi spuntarla sul terreno elettorale con il corpo elettorale che abbiamo, con i compagni che abbiamo e con la situazione che c'è? Era giusto dare il voto alle donne?», dando per scontata l'influenza della Chiesa sulle masse femminili.

${ }^{6}$ ETTORRE, Lorenzo, Il Pci e il Concilio Vaticano II, cit., p. 112. Cfr. anche ID., «Il Concilio Vaticano II nella stampa comunista italiana (1959-1965)», in Diacronie. Studi di Storia Contemporanea. Le sembianze di Clio: approcci alla storia, 8, 4/2011,

URL: < http://www.studistorici.com/2011/10/29/ettorre_numero_8/ > [consultato il 24 aprile 2015]. 
Certo, rimane l'interesse prevalentemente strumentale e politico, dettato dalla speranza che il Concilio, costringendo a far i conti con la "modernità", potesse incrinare la compattezza della Chiesa, nonché il suo appoggio alla DC e pertanto contribuisse a impedire il perpetuarsi dell'isolamento del Partito Comunista.

A parte i singoli punti - compresi i giudizi su Paolo VI, sui quali si rinvia a una diretta lettura del volume - bisogna soffermarsi su quella che forse è la conclusione principale del nostro autore. Come già segnalato dal sottotitolo del libro, infatti, Ettorre ritiene che grazie al Concilio Vaticano II i comunisti furono sollecitati a comprendere che dovevano ormai confrontarsi con una Chiesa pluralistica e ricca di fermenti, che non era più riduttivamente un mero bastione della reazione e del governo democristiano. In tal modo si aprivano spazi per un'apertura intellettuale più ampia verso l'intera "questione" cattolica o, se si preferisce, si poneva qualche premessa per la riconsiderazione della religione, utilizzando categorie concettuali e strumenti di analisi più adeguati rispetto a quelli della tradizione del partito. Di conseguenza, conclude Ettorre, per il PCI il Concilio rappresentò anche «un potente e inaspettato acceleratore funzionale alla elaborazione di una rinnovata strategia politica»7. In altre parole: la preminenza accordata da Togliatti nel dopoguerra al rapporto con la DC si attenuava, mentre cresceva quella per la Chiesa. Parallelamente si affinava l'attenzione per le crescenti espressioni di dissenso intraecclesiale, non più viste come irrilevanti $\mathrm{o}$ al più come potenziali cunei da porre tra vertice e base, bensì come realtà dotate di un valore in sé.

Un'ulteriore segnalazione va fatta per la buona documentazione archivistica che, di tanto in tanto, l'autore impiega per arricchire la sua narrazione. Degni di nota, infatti, sono i riferimenti che Ettorre fa più volte al ruolo giocato da Libero Pierantozzi - che segue per «Rinascita» i lavori del Concilio - nel fornire materiale e suggerimenti ai vertici del partito e soprattutto al segretario Luigi Longo, con la costante presenza di un intellettuale di spessore come Lucio Lombardo Radice.

Per tutta questa seconda parte del libro sarebbe stato però interessante verificare le eventuali discussioni avvenute nel PCI a livello di dirigenza, integrando i sondaggi archivistici effettuati dall'autore sullo svolgimento del $\mathrm{X}$ congresso e sulla corrispondenza di Longo. Non sono in grado di affermare se discussioni ci furono e di che livello, ma posso portare un esempio successivo di un paio di anni. Quando Paolo VI pubblicò l'enciclica Populorum progressio (26 marzo 1967), la direzione del PCI si sentì in dovere di dedicare un'intera riunione all'argomento. Giancarlo Pajetta presentò il tema, sostenendo che si trattava di un «documento politico», che rappresentava un

7 ETTORRE, Lorenzo, Il Pci e il Concilio Vaticano II, cit., p. 184. 
«momento di autonomia rispetto alle forze sociali con cui la Chiesa in questo periodo è stata collegata. Più che per il passato, inoltre, la Chiesa sente gli impulsi che vengono dal basso». La valutazione positiva continuava: «Siamo molto più avanti rispetto alla politica socialdemocratica tra le due guerre», affermò Pajetta, invitando a dare «la massima attenzione» all'enciclica, nella quale si coglieva con soddisfazione l'inesistenza di argomentazioni anticomuniste. Insomma, le soluzioni della Populorum progressio erano «fortemente riformiste e empiriche». Nella discussione intervennero, tra gli altri, anche Natta, Napolitano, Sereni e Ingrao. Il futuro Capo dello Stato osservò con soddisfazione che il partito era stato pronto a cogliere l'importanza dell'enciclica, mentre Sereni - ma non solo lui, per la verità - colse bene i tratti di novità del testo di Paolo VI:

Quel che è assolutamente nuovo, anche rispetto a Giovanni XXIII, è il linguaggio. È il linguaggio della nuova sociologia francese, in gran parte di estrazione americana. Non più linguaggio religioso, ma linguaggio da organizzazione politica. C'è il tentativo di presentarsi come i portatori di una ideologia egemonica per il terzo mondo e i paesi di capitalismo avanzato, diverso da quello di Giovanni XXIII teso a sottolineare il momento del dialogo con il mondo socialista.

Resta però che la capacità liberatrice di questa enciclica, per forze cattoliche, è molto grande. È la prima volta che assistiamo a una critica strutturale del capitalismo e del sistema fondato sul profitto. Di conseguenza anche la limitatezza delle soluzioni proposte acquista un significato diverso ${ }^{8}$.

Questi riferimenti e queste citazioni cadono naturalmente al di là dei limiti temporali fissati da Ettorre per il suo libro. Essi servono tuttavia a mostrare quanto interessante lavoro attenda ancora gli storici, nella tacita speranza di poter esaminare quanto prima anche le carte più riservate della controparte dei comunisti, ovvero la Chiesa cattolica.

${ }^{8}$ Fondazione Gramsci, Archivio del Partito Comunista, Direzione, Verbali, riunione del 5 aprile 1967. 


\section{* L'autore}

Giorgio Vecchio è nato a Como nel 1950, laureato in Scienze Politiche presso l'Università Cattolica di Milano nel 1973. Professore ordinario di Storia Contemporanea presso l'Università di Parma. È presidente del Comitato scientifico della Fondazione Don Primo Mazzolari (Bozzolo, Mantova). Dirige la collana "Edizione critica delle opere di don Primo Mazzolari" presso le edizioni Dehoniane di Bologna. È membro del comitato scientifico dei seguenti istituti di ricerca: Istituto per la storia dell'Azione Cattolica e del Movimento cattolico in Italia 'Paolo VI' (Roma); Istituto Alcide Cervi per la storia dell'agricoltura, dei movimenti contadini, dell'antifascismo e della Resistenza nelle campagne (Gattatico, Reggio Emilia). I suoi interessi vertono sulla storia del movimento cattolico italiano ed europeo, dei movimenti pacifisti, della politica e della società italiana, dei genocidi e della Shoah.

URL: < http://www.studistorici.com/progett/autori/\#Vecchio >

\section{Per citare questo articolo:}

VECCHIO, Giorgio, «Recensione: Lorenzo ETTORRE, II Pci e il Concilio Vaticano II. Dal partito dei cattolici al cattolicesimo, Roma, Studium, 2014, 188 pp.», Diacronie. Studi di Storia Contemporanea : Costruire. Rappresentazioni, relazioni, comunità, 29/06/2015,

URL:< http://www.studistorici.com/2015/06/29/vecchio_numero_22/ >

Diacronie Studi di Storia Contemporanea $\}$ www.diacronie.it

Risorsa digitale indipendente a carattere storiografico. Uscita trimestrale.

redazione.diacronie@hotmail.it

Comitato di redazione: Jacopo Bassi - Luca Bufarale - Elisa Grandi - Deborah Paci - Fausto Pietrancosta - Matteo Tomasoni - Luca Zuccolo 